OPEN ACCESS

Edited by:

Kirankumar Mysore,

The Samuel Roberts Noble

Foundation, USA

Reviewed by:

Lindsay Renee Triplett,

Connecticut Agricultural Experiment

Station, USA

Ana Maria Bocsanczy,

University of Florida, USA

${ }^{*}$ Correspondence:

Mathilde Fagard

mathilde.fagard@versailles.inra.fr

Specialty section:

This article was submitted to

Plant Biotic Interactions,

a section of the journal

Frontiers in Plant Science

Received: 13 January 2016

Accepted: 07 April 2016

Published: 26 April 2016

Citation:

Launay $A$, Patrit $O$, Wénès $E$ and Fagard $M$ (2016) DspA/E

Contributes to Apoplastic Accumulation of ROS in Non-host A. thaliana. Front. Plant Sci. 7:545.

doi: $10.3389 /$ fpls.2016.00545

\section{DspA/E Contributes to Apoplastic Accumulation of ROS in Non-host A. thaliana}

\author{
Alban Launay ${ }^{1,2}$, Oriane Patrit ${ }^{3}$, Estelle Wénès ${ }^{1}$ and Mathilde Fagard ${ }^{1 *}$ \\ ${ }^{1}$ CNRS 3559, Institut Jean-Pierre Bourgin, INRA, AgroParisTech, ERL, Université Paris-Saclay, Versailles, France, \\ ${ }^{2}$ Université Paris-Sud-Université Paris-Saclay, Orsay, France, ${ }^{3}$ AgroParisTech, Paris, France
}

The bacterium Erwinia amylovora is responsible for the fire blight disease of Maleae, which provokes necrotic symptoms on aerial parts. The pathogenicity of this bacterium in hosts relies on its type three-secretion system (T3SS), a molecular syringe that allows the bacterium to inject effectors into the plant cell. E. amylovora-triggered disease in host plants is associated with the T3SS-dependent production of reactive oxygen species (ROS), although ROS are generally associated with resistance in other pathosystems. We showed previously that E. amylovora can multiply transiently in the non-host plant Arabidopsis thaliana and that a T3SS-dependent production of intracellular ROS occurs during this interaction. In the present work we characterize the localization and source of hydrogen peroxide accumulation following $E$. amylovora infection. Transmission electron microscope (TEM) analysis of infected tissues showed that hydrogen peroxide accumulation occurs in the cytosol, plastids, peroxisomes, and mitochondria as well as in the apoplast. Furthermore, TEM analysis showed that an E. amylovora dspAVE-deficient strain does not induce hydrogen peroxide accumulation in the apoplast. Consistently, a transgenic line expressing DspA/E accumulated ROS in the apoplast. The NADPH oxidase-deficient rbohD mutant showed a very strong reduction in hydrogen peroxide accumulation in response to $E$. amylovora inoculation. However, we did not find an increase in bacterial titers of $E$. amylovora in the rbohD mutant and the rbohD mutation did not suppress the toxicity of DspA/E when introgressed into a DspA/E-expressing transgenic line. Co-inoculation of $E$. amylovora with cycloheximide $(\mathrm{CHX})$, which we found previously to suppress callose deposition and allow strong multiplication of $E$. amylovora in $A$. thaliana leaves, led to a strong reduction of apoplastic ROS accumulation but did not affect intracellular ROS. Our data strongly suggest that apoplastic ROS accumulation is one layer of the non-host defense response triggered by the type three effector (T3E) DspA/E, together with callose deposition.

Keywords: non-host, Erwinia amylovora, Arabidopsis thaliana, ROS, DspA/E

\section{INTRODUCTION}

One of the most common and earliest responses of plants to pathogens is reactive oxygen species (ROS) production (Lamb and Dixon, 1997). In the case of biotrophic pathogens, ROS production is usually associated with defense (Torres et al., 2002) but in the case of necrotrophic pathogens the role of ROS production is less clear. For example, the necrotrophic fungus Botrytis cinerea 
induces the production of hydrogen peroxide $\left(\mathrm{H}_{2} \mathrm{O}_{2}\right)$ that is associated with disease (van Baarlen et al., 2007). It was also shown that the NbrbohB-dependent production of $\mathrm{H}_{2} \mathrm{O}_{2}$ following infection by $B$. cinerea contributes to lesion development in Nicotiana benthamiana (Asai and Yoshioka, 2009). In the case of the necrotrophic bacterium Dickeya dadantii, strong oxidative stress was observed in Arabidopsis thaliana and it was shown that this accumulation contributes to defense (Fagard et al., 2007). In A. thaliana, RbohD was found to be the main source of ROS production to the avirulent strain of Pseudomonas syringae avrRpm1 (Torres et al., 2002) and to the bacterial necrotroph $D$. dadantii (Fagard et al., 2007). The current knowledge on RbohD, which encodes an NADPH oxidase, has been recently reviewed (Marino et al., 2012).

Non-host resistance is a widespread process in plants that corresponds to the resistance of a plant species to all the isolates of a given pathogen species that is pathogenic on other plant species (Schulze-Lefert and Panstruga, 2011). Indeed, most plants are resistant to most pathogens and disease remains the exception. While non-host resistance of plants to pathogenic fungi has attracted some attention, only a few studies have focused on nonhost resistance of plants to bacterial pathogens. Several examples of the role of ROS accumulation during non-host interactions have been described in the literature. For example, Senthil-Kumar and Mysore showed in 2012 that A. thaliana deficient mutants Soat and $p 5 c d h$, which are deficient for ROS accumulation, are compromised in the non-host resistance to P. syringae pv. tabaci. Likewise, suppression of glycolate oxidase genes compromised $\mathrm{H}_{2} \mathrm{O}_{2}$ accumulation in $A$. thaliana in response to $P$. syringae pv. tabaci which is associated with more bacterial multiplication (Rojas et al., 2012). Another example is the non-host interaction between Pinus pinaster and B. cinerea in which a biphasic ROS accumulation is associated to a hypersensitive response (Azevedo et al., 2008).

Erwinia amylovora is the causal agent of bacterial fire blight, which affects rosaceae plants mainly of the pyreae tribe. The pathogenicity of E. amylovora depends mainly on the type three secretion system (T3SS) which secretes and injects type three effectors (T3Es) inside the plant cell; among them, the T3E $\mathrm{DspA} / \mathrm{E}$ plays a major role as a $d s p A / E$ deficient mutant is nonpathogenic (Barny et al., 1990). In host plants, E. amylovora induces ROS accumulation and induces the activity of ROS detoxifying enzymes such as glutathion-S-transferases (Venisse et al., 2003). ROS accumulation in host leaves is T3SS-dependent as a non-pathogenic type three secretion mutant does not induce ROS accumulation (Venisse et al., 2001). E. amylovora was found to be approximately 10 times less susceptible to oxidative stress in vitro than the biotrophic bacterium $P$. syringae pv. tomato, leading the authors to hypothesize that oxidative stress induced by E. amylovora was required for cell death induction and disease (Venisse et al., 2003).

We showed previously that in non-host $A$. thaliana, E. amylovora is able to multiply transiently and induce necrotic symptoms but that $A$. thaliana mounts an efficient defense, which leads to a decrease in bacterial titers after $48 \mathrm{~h}$ (Barny et al., 2008; Degrave et al., 2008; Moreau et al., 2012). Multiplication of E. amylovora in A. thaliana leaves is dependent on the DspA/E effector protein and associated with the production of necrotic symptoms, as in host plants. Indeed, transgenic plants expressing DspA/E, allowed a $d s p A / E$-deficient mutant to multiply in planta (Degrave et al., 2013). Although, non-host resistance of A. thaliana toward E. amylovora has not been elucidated, our previous work showed that it requires an activation of plant defense since co-inoculation with cycloheximide (CHX), a repressor of protein synthesis, led to an important multiplication of E. amylovora in A. thaliana (Moreau et al., 2012).

In the present work, we analyze in detail ROS production during the non-host interaction between E. amylovora and A. thaliana and we analyze the role of DspA/E in this ROS production. Our results demonstrate that E. amylovora triggers DspA/E-independent intracellular $\mathrm{H}_{2} \mathrm{O}_{2}$ accumulation and DspA/E-dependent apoplast accumulation of $\mathrm{H}_{2} \mathrm{O}_{2}$. We show that $\mathrm{RbohD}$ is involved in $\mathrm{H}_{2} \mathrm{O}_{2}$ accumulation and suppression of necrosis but we did not find any evidence that it was required for resistance. Co-inoculation with cycloheximide $(\mathrm{CHX})$, previously shown to allow a strong increase in bacterial titers, did not affect intracellular ROS accumulation but strongly reduced apoplastic ROS accumulation. Altogether, our results suggest a role for apoplastic $\mathrm{H}_{2} \mathrm{O}_{2}$ accumulation in non-host resistance.

\section{MATERIALS AND METHODS}

\section{Plant Lines and Bacterial Strains Used in This Study}

Seeds of $A$. thaliana Col-0 were obtained from the INRA Versailles collection. Plants were grown for 5 weeks in soil and were subjected to an $8 \mathrm{~h}$-light and $16 \mathrm{~h}$-dark cycle at $21^{\circ} \mathrm{C}$ (day) $/ 18^{\circ} \mathrm{C}$ (night) with $65 \%$ relative humidity. Seeds of the rbohD mutant (Torres et al., 2002) were obtained from J. Dangl (Chapel Hill, NC, USA). The double rbohD/13-1-2 line was obtained in the laboratory by crossing.

Erwinia amylovora wild-type strain CFBP1430 (Ea), T3SSdeficient strain CFBP6023 (tts) were obtained from the Collection Française des Bactéries Phytopathogènes (CFBP). The E. amylovora $d s p A / E$-deficient strain $(d s p A / E)$ is described in Degrave et al. (2013). For plant inoculation, a liquid pre-culture of each strain was plated on $\mathrm{LB}$ and grown overnight at $28^{\circ} \mathrm{C}$.

The 7-2-1 GFP-expressing $A$. thaliana transgenic line and the 13-1-2 $d s p A / E$-expressing $A$. thaliana transgenic line are described in Degrave et al. (2013). For root growth measurement, estradiol treatment was performed on 6-days-old seedlings grown on 1/5th Murashige and Skoog supplemented with $1 \%$ sucrose. A stock solution of estradiol was initially prepared in Dimethyl sulfoxide (DMSO) at $20 \mathrm{mM}$ and stored at $-20^{\circ} \mathrm{C}$. The root of each seedling was treated with a drop of $200 \mu \mathrm{L}$ of an estradiol solution (diluted to the indicated concentration in water), or mock-treated for $10 \mathrm{~min}$ and transferred to a growth chamber (16 h day/8 h night). Pictures were taken $24 \mathrm{~h}$ after treatment and root length measurement was performed using the ImageJ software. For ROS analysis, a $5 \mu \mathrm{M}$ estradiol solution was infiltrated into leaves of 5-weeks-old rosette that were sampled and stained at the indicated time point. 


\section{Bacterial Infection}

A bacterial suspension of E. amylovora with an OD of 0,1 $\left(10^{7} \mathrm{CFU} / \mathrm{mL}\right)$ in water was syringe-inoculated into 5 -weeks-old plants. For symptom intensity and bacterial count the procedure is described in Degrave et al. (2008). For CHX treatment, E. amylovora was co-inoculated with the translation inhibitor cycloheximide (Sigma) at $4 \mu \mathrm{g} / \mathrm{ml}$ as described in Moreau et al. (2012).

\section{Detection of ROS}

$2^{\prime}, 7^{\prime}$-Dichlorofluorescein diacetate (DCFH-DA) staining was used to detect intracellular hydrogen peroxide. A $30 \mathrm{mM}$ DCFHDA (Sigma) solution was prepared in DMSO and diluted 100 times in deionized water. Inoculated or mock-treated leaves were collected $16 \mathrm{~h}$ post-inoculation (hpi) and vacuum-infiltrated with DCFH-DA. Leaves were immediately put on a microscope slide and fluorescence emission was observed under a binocular magnifier with a GFP filter $(510 \mathrm{~nm})$.

Diaminobenzidine (DAB) staining was used to detect intraand extracellular hydrogen peroxide $\left(\mathrm{H}_{2} \mathrm{O}_{2}\right)$. A $5 \mathrm{mg} / \mathrm{mL}$ DAB (Sigma) stock solution $\mathrm{pH}$ 3,7 was prepared with deionized water and diluted to a final concentration of $1 \mathrm{mg} / \mathrm{ml}$. Five-weeksold leaves were collected $2 \mathrm{~h}$ after inoculation (as indicated) and vacuum infiltrated with the DAB solution. Leaves were then placed in a wet Petri dish overnight and the staining was stopped at $16 \mathrm{hpi}$ by placing the leaves in ethanol to discolor them. Once all chlorophyll was removed, leaves were mounted in water between a microscopic slide and a cover glass and observed with a Nikon Microphot FXA photonic microscope at X400 magnification.

\section{Transmission Electron Microscopy (TEM) Observations}

For the transmission electron microscopy (TEM) observations, pieces of 0,1 by $0,3 \mathrm{~mm}$ were sampled from mock-treated or inoculated leaves as described. Samples were incubated in $5 \mathrm{mM}$ cerium chloride $\left(\mathrm{CeCl}_{3}\right)$ in MOPS buffer $50 \mathrm{mM} \mathrm{pH} 7.2$ during $1 \mathrm{~h}$ at room temperature. Samples were fixed in $0,1 \mathrm{M}$ cacodylate buffer $(1,5 \%$ paraformaldehyde and $2 \%$ glutaraldehyde $\mathrm{pH} 7,2)$ during $4 \mathrm{~h}$ under agitation with a $1 \mathrm{~min}$ vacuum infiltration every hour. Samples were then rinsed four times in cacodylate buffer during 15 min under agitation and placed in the contrast solution (cacodylate $0.1 \mathrm{M} \mathrm{pH} \mathrm{7.2,} \mathrm{Osmium} \mathrm{Tetroxide)} \mathrm{during}$ $1 \mathrm{~h}$. Samples were rinsed three times with deionized water during $10 \mathrm{~min}$ under agitation and subjected to increasing dehydration with ethanol 25, 50, 70, 85\% during $30 \mathrm{~min}$ and finally in ethanol $90 \%$ overnight at $4^{\circ} \mathrm{C}$. Samples were then subjected to absolute ethanol three times during $30 \mathrm{~min}$. Samples were treated with $50 \%$ absolute ethanol/50\% propylene oxide during 30 min under agitation followed by three $100 \%$ propylene oxide baths. Embedding consisted in an overnight bath of 2/3 propylene oxide, 1/3 Epon-araldite (Electron Microscopy Sciences) at room temperature and a second bath with $1 / 3$ propylene oxide, 2/3 Epon-araldite applied for 9 h. Following propylene oxide evaporation, two baths with pure Epon-araldite resin were applied. Samples were placed in silicon flat molds and resin was polymerized at $60^{\circ} \mathrm{C}$ for $48 \mathrm{~h}$. Semi-thick sections were made with a razor blade and a Histome diamond knife for quality control of each sample (stained with $0,1 \%$ toluidine blue in water). Finally, samples were cut with an ultra-thin section diamond knife $(70 \mathrm{~nm})$. Cut samples were collected on electron microscope grids (200 mesh) for further observation on a Zeiss EM912 OMEGA electron microscope at a magnification of 10000 to $50000 \mathrm{X}$.

\section{Statistical Analysis and ImageJ Software}

The Mann and Whitney test was selected because we compared quantitative values with two independent conditions with less than 30 samples per condition. Intensity of DAB staining or DCFH-DA fluorescence was quantified using the ImageJ software (version 1.46r). The intensity was measured in a square section of the half-blade that was inoculated using the "Integrated density" (intdens) function of the software. This operation was repeated on 5-10 leaves per condition depending on the experiment. The results presented correspond to the mean intensity for each condition for a given experiment. All experiments were performed three times and a representative experiment is showed. The significant differences presented in the figures were found in each independent experiment.

\section{RESULTS}

\section{Erwinia amylovora Induces Accumulation of Intracellular and Extracellular Hydrogen Peroxide}

We showed previously that E. amylovora induces the accumulation of intracellular ROS detected by the fluorescent probe DCFH-DA (Degrave et al., 2008; Figure 1A), which allows detection of hydrogen peroxide. In order to determine the localization of $\mathrm{H}_{2} \mathrm{O}_{2}$ accumulation in response to E. amylovora inoculation, we used diaminobenzidine (DAB), which forms a brown precipitate in the presence of $\mathrm{H}_{2} \mathrm{O}_{2}$. Five-weeks-old A. thaliana rosette leaves were infiltrated with $E$. amylovora and the accumulation of $\mathrm{H}_{2} \mathrm{O}_{2}$ was determined at $16 \mathrm{hpi}$. Most E. amylovora-infected leaves (84\%) displayed strong DAB staining (Figure 1B). In general, mock-inoculated leaves displayed no DAB staining but a small proportion of leaves (18\%) displayed slight DAB staining (Figure 1B). We then observed the DAB-stained leaves by photon microscopy, which revealed that mock-infected leaves displayed only very slight diffuse brown staining while wild-type E. amylovora-infected leaves displayed distinct brown staining in groups of cells in both the palisade and the spongy parenchyma (Figure 1C). The brown precipitate accumulated both inside the cells and at their periphery. In the groups of stained cells, numerous round organelles were distinctly stained with brown precipitate (Figure 1C), the number and size of which suggest that they could be plastids.

Altogether, our results confirm that infection by E. amylovora of the non-host plant $A$. thaliana leads to hydrogen peroxide accumulation in numerous round organelles as well as at the 
A

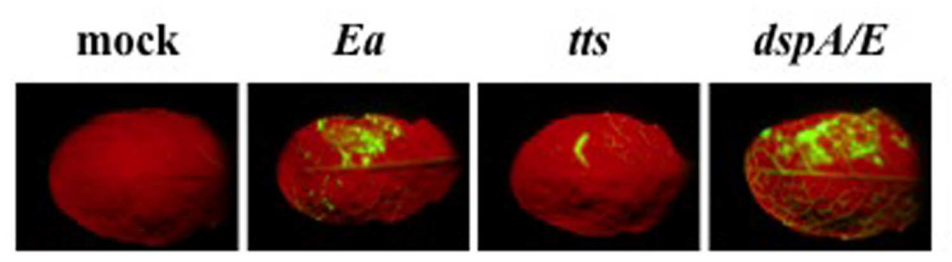

B

\section{mock}

$E a$

tts
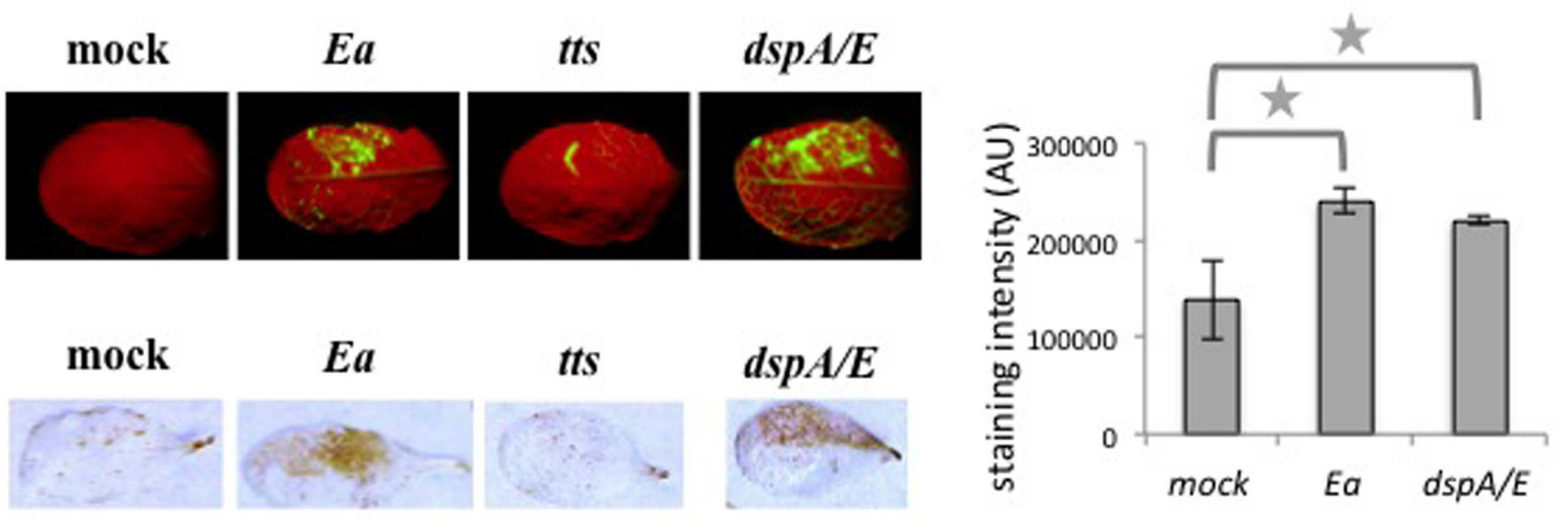

C

mock
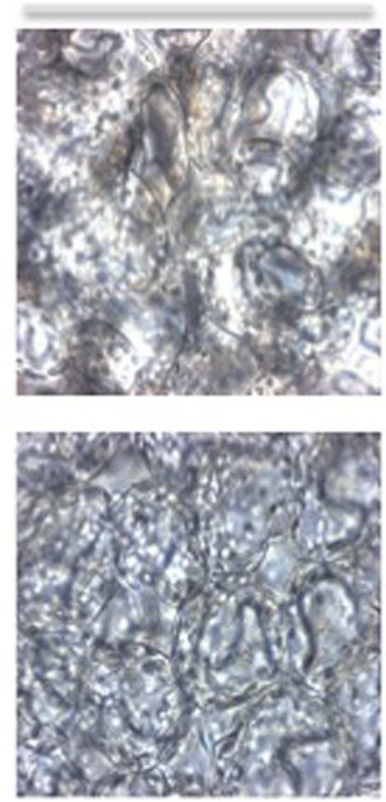

$E a$
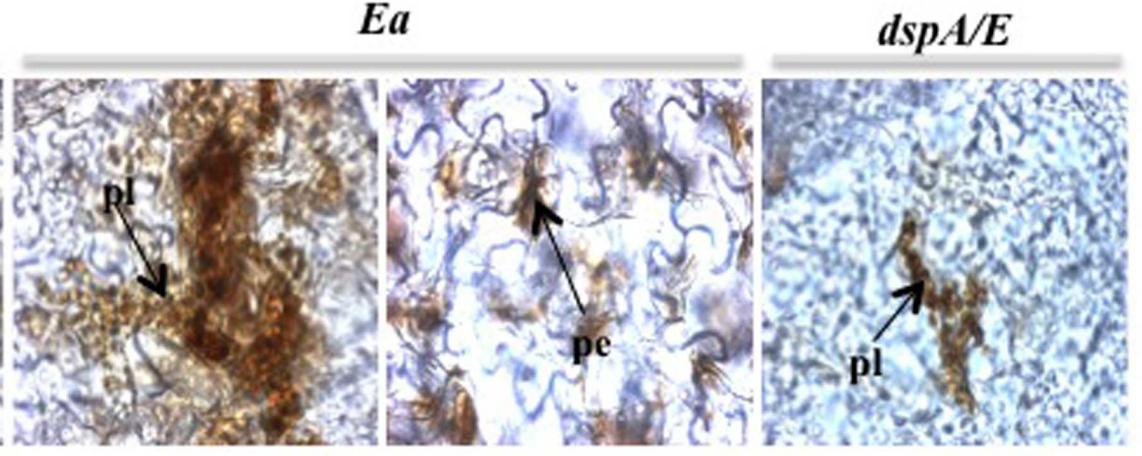

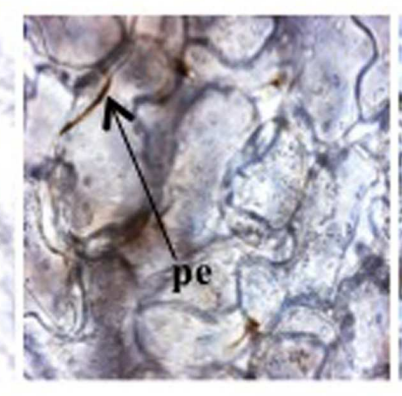

FIGURE 1 | Erwinia amylovora induces accumulation of $\mathbf{H}_{\mathbf{2}} \mathbf{O}_{\mathbf{2}}$. (A-C) Leaves of 5-weeks-old plants were infiltrated on the upper half of the leaf blade with water (mock), E. amylovora (Ea) wild-type strain, T3SS-deficient mutant (tts) or dspAVE mutant (dspA/E). (A) Leaves stained with DCFH-DA show intracellular $\mathrm{H}_{2} \mathrm{O}_{2}$ accumulation in green. (B) Brown stains indicate accumulation of $\mathrm{H}_{2} \mathrm{O}_{2}$ in leaves stained with DAB, the graph to the right shows DAB staining intensity. The stars indicate a significant difference according to Mann and Whitney's statistical test $(0<0.05)$. (C) Localization of $\mathrm{H}_{2} \mathrm{O}_{2}$ deposits in DAB-stained leaves observed under a photon microscope. Bar: $50 \mu \mathrm{m}$; pl: plastid; pe: staining at the periphery of the cell. Similar results were obtained for three independent experiments. Top panel: palissadic parenchyma; bottom panel: spongy parenchyma.

periphery of cells, however due to the weak resolution of the light microscope technique, the precise localization could not be determined.

\section{A dspA/E-Defective E. amylovora Mutant Can Induce ROS Accumulation in}

\section{A. thaliana}

Our previous work shows that during the interaction between A. thaliana and E. amylovora, the T3SS is responsible for intracellular ROS accumulation detected by DCFH-DA (Degrave et al., 2008; Figure 1A). Among the known effectors to be injected by E. amylovora inside plant cells, DspA/E plays a crucial role during disease-related ROS accumulation on host plants (Venisse et al., 2003) and during the interaction with A. thaliana (Degrave et al., 2013). In order to determine the role of DspA/E in ROS production, we analyzed ROS accumulation following inoculation with a $d s p A / E$-defective mutant using DCFH-DA and DAB staining. We also used a T3SS-defective strain for comparison. Consistent with our previous results, the T3SSdefective strain did not induce ROS accumulation detected by DCFH-DA (Figure 1A). Consistently, leaves inoculated with 
a T3SS-defective strain (Figure 1B) did not accumulate ROS detected by DAB staining.

In contrast, our results show that leaves inoculated with a $d s p A / E$-defective mutant accumulate both DCFH-DA and DAB-detectable ROS (Figures 1A,B). At the tissue level, leaves inoculated with a $d s p A / E$-defective mutant accumulated ROS in both palisade and spongy parenchyma and intracellular staining could be observed, in particular in the round organelles (Figure 1C).

Altogether, our results show that a $d s p A / E$-deficient strain, contrary to a T3SS-deficient strain, remains able to trigger $\mathrm{H}_{2} \mathrm{O}_{2}$ accumulation in A. thaliana, indicating that other elements of the T3SS than DspA/E are involved in ROS accumulation during non-host resistance.

\section{E. amylovora Induces Strong $\mathrm{H}_{2} \mathrm{O}_{2}$ Accumulation in the Apoplast}

In order to determine more precisely the localization of hydrogen peroxide accumulation, we decided to use $\mathrm{CeCl}_{3}$ staining which forms electron-dense deposits in the presence of hydrogen peroxide. Five-weeks-old A. thaliana plants were mock-treated or E. amylovora-inoculated, sampled 24 hpi and ultra-thin sections of $\mathrm{CeCl}_{3}$-stained leaves were observed with an electron microscope (Figure 2). The staining revealed that in E. amylovora-infected leaves hydrogen peroxide could be detected in all visible organelles: mitochondria, peroxisomes, chloroplasts as well as in the cytoplasm (Figure 2A). In addition, a very strong electron-dense staining was observed at the periphery of the cells, most likely in the apoplast, which was not observed in mock-inoculated leaves. Strong electron-dense staining was also observed around the bacteria, which could be seen in the apoplastic space or attached to the cell wall (Figure 2B).

Our results show that E. amylovora infection of non-host A. thaliana elicits the accumulation of hydrogen peroxide in different cellular compartments including chloroplasts, and reveals that $E$. amylovora triggers a strong hydrogen peroxide accumulation in the apoplast.

\section{DspA/E is Necessary for $\mathrm{H}_{2} \mathrm{O}_{2}$ Accumulation in the Apoplast}

In order to determine the role of the T3E DspA/E in the triggering of ROS localized in the apoplast, we observed with TEM ultrathin sections of $\mathrm{CeCl}_{3}$-stained leaves inoculated with the $d s p A / E$ deficient strain. Our results indicate that intracellular ROS accumulated as in response to the wild-type strain (Figure 2A), which is consistent with our observations using $\mathrm{DAB}$ and DCFH-DA staining (Figures 1A-C). On the other hand, the strong ROS accumulation observed in the apoplast in leaves inoculated with the wild-type strain of E. amylovora was not
A

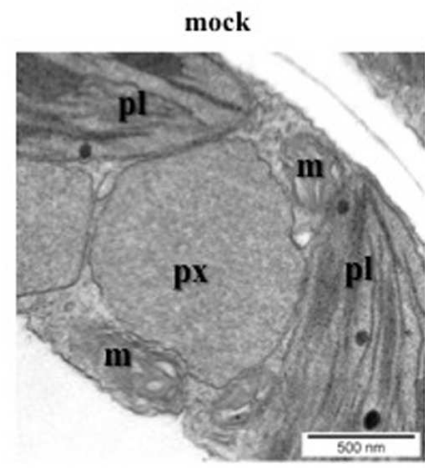

B

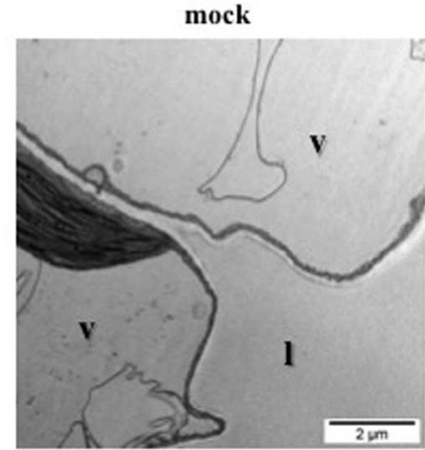

Ea

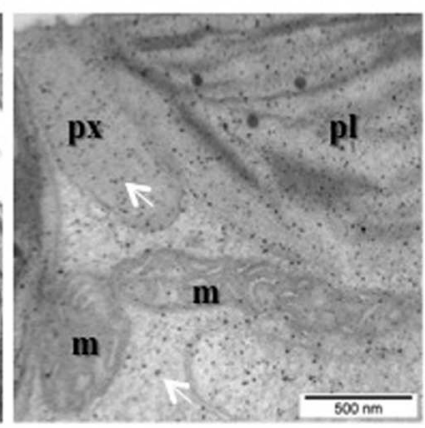

Ea

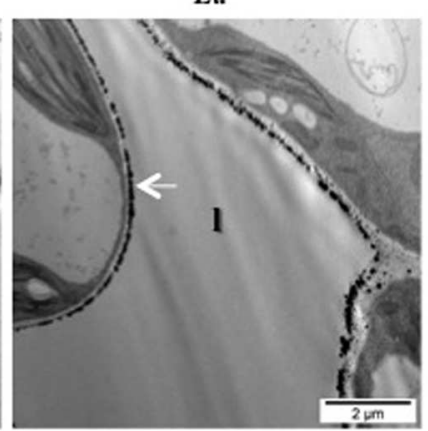

$d s p A / E$

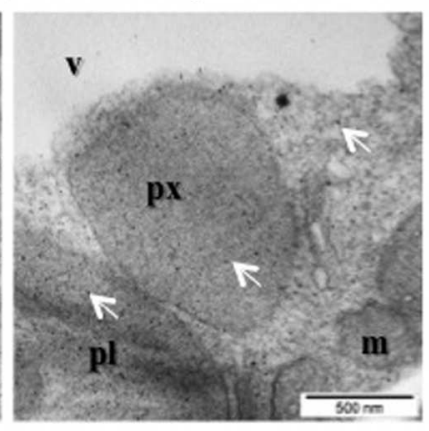

$d s p A / E$

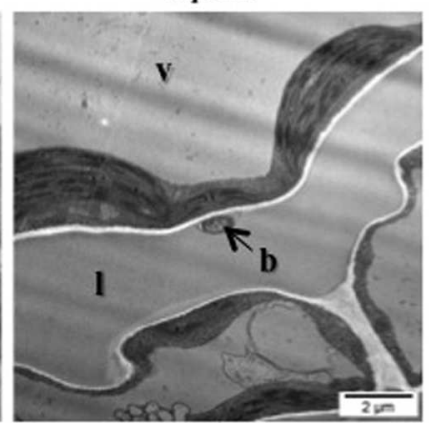

FIGURE 2 | Erwinia amylovora induces strong $\mathrm{H}_{2} \mathrm{O}_{2}$ accumulation in the cytoplasm and in the apoplast. (A,B) TEM observation of CeCl 3 -stained leaves. Black spots indicate $\mathrm{H}_{2} \mathrm{O}_{2}$ accumulation stained by $\mathrm{CeCl}_{3}$ (white arrows). Leaves of 5-weeks-old plants were infiltrated with water (mock), wild-type E. amylovora ( $E a)$, or with the $d s p A / E$ mutant $(d s p A / E)$ and collected 16 hpi. (A) Bar: $500 \mathrm{~nm}$. (B) Bar: $2 \mu \mathrm{m}$. b: bacterium, l: lacuna, m: mitochondrion, pl: plastid, px: peroxisome, v: vacuole. 
observed in leaves inoculated with the $d s p A / E$-deficient strain (Figure 2B).

To confirm the role of DspA/E in the triggering of ROS in the apoplast, we used the 13-1-2 transgenic line expressing DspA/E under the control of an estradiol-inducible promoter that we described previously (Degrave et al., 2013). Ultrathin sections of $\mathrm{CeCl}_{3}$-stained leaves were observed by TEM (Figure 3A). Control plants (7-2-1) did not show any staining whether treated with estradiol or not (Figure 3A). In contrast, plants from the 131-2 line, bearing the bacterial $D s p A / E$ gene, treated with estradiol to induce $D s p A / E$ expression displayed staining in portions of the apoplast but no staining in the cytoplasm (Figure 3A).
Mock-treated 13-1-2 plants also displayed slight staining in the apoplast which is consistent with low expression of $D s p A / E$ in mock-treated 13-1-2 plants as we showed previously (Degrave et al., 2013).

In order to confirm the specific role of DspA/E in triggering only apoplastic ROS, we stained the transgenic lines described above with DCFH-DA. As expected, the 7-2-1 control line did not show significant fluorescence while leaves inoculated with E. amylovora, used as a positive control, were clearly fluorescent (Figure 3B). The 13-1-2 line did not show significant fluorescence whether $D s p A / E$ expression was induced with estradiol or not (Figure 3B). The intensity of fluorescence was quantified using
A

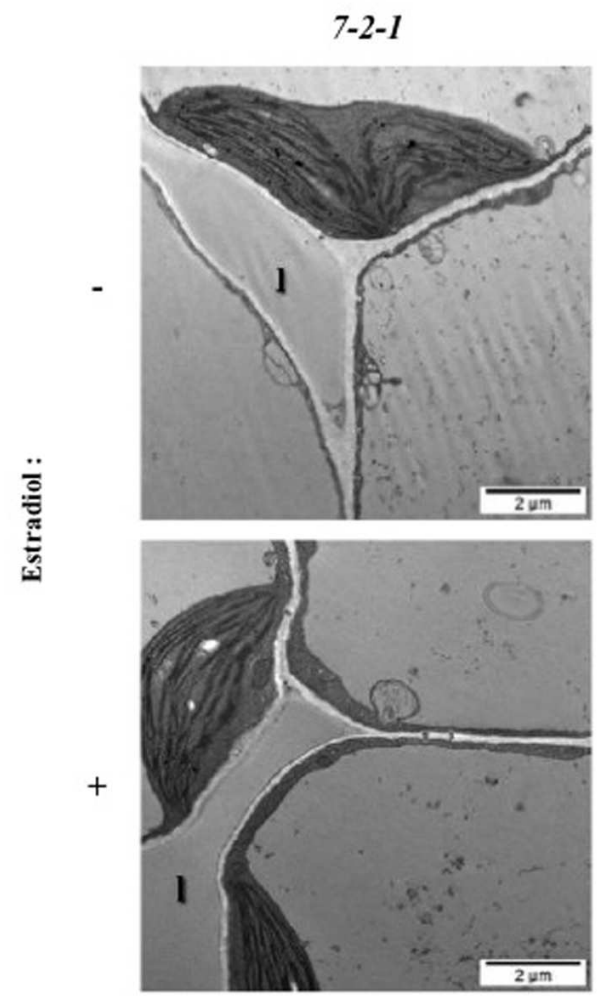

B

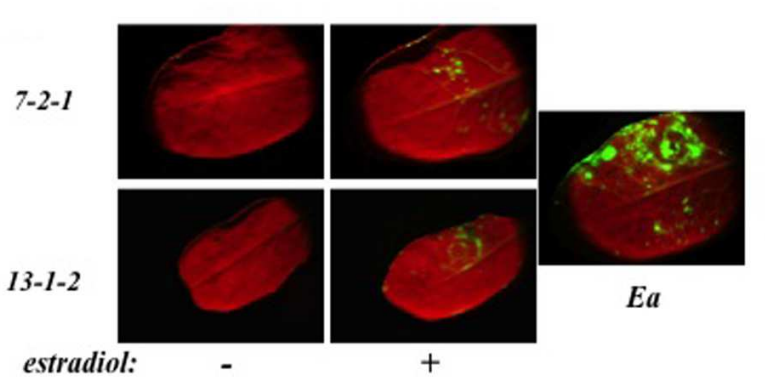

\section{3-1-2}
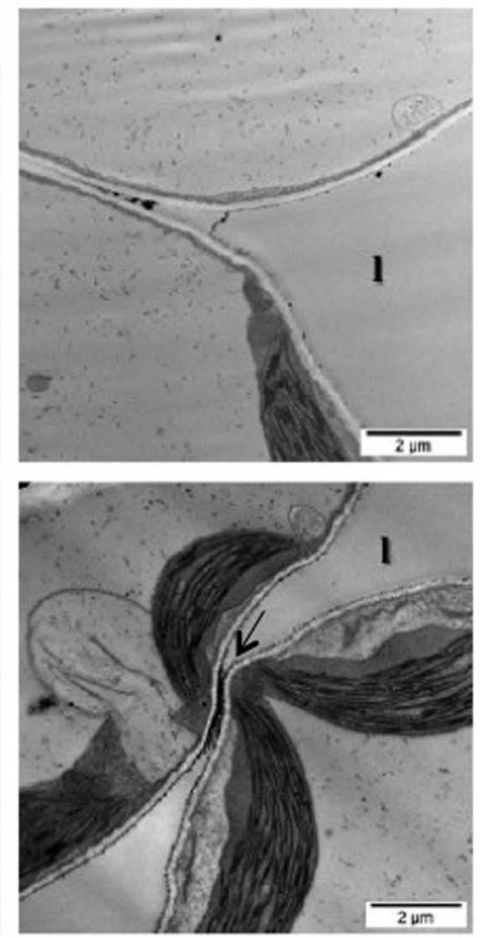

C

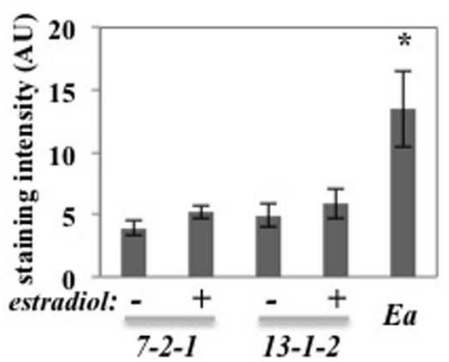

FIGURE 3 | DspA/E expressed in planta induces $\mathbf{H}_{\mathbf{2}} \mathbf{O}_{\mathbf{2}}$ accumulation in the apoplast. (A,B) Leaves of 5-weeks-old plants were infiltrated with water (-) or a $5 \mu \mathrm{M}$ estradiol solution (+) and collected 16 hpi. (A) TEM observation of $\mathrm{CeCl}_{3}$-stained leaves. Black spots indicate $\mathrm{H}_{2} \mathrm{O}_{2}$ accumulation stained by CeCl 3 (arrow). Bar: $2 \mu \mathrm{m}$. I: lacuna. (B) DCFH-DA staining shows intracellular $\mathrm{H}_{2} \mathrm{O}_{2}$ accumulation in green. E. amylovora (Ea) inoculation was used as a positive control for DCFH-DA staining. (C) Mean DCFH-DA staining intensity, which was determined in 5-10 leaves per condition using the Image S Software. The asterisks indicate a significant difference according to Mann and Whitney's statistical test $(p<0.05)$. 
A
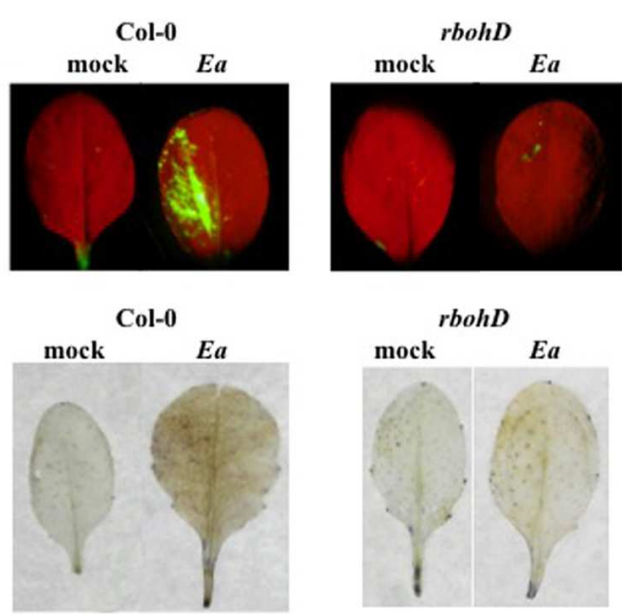

B

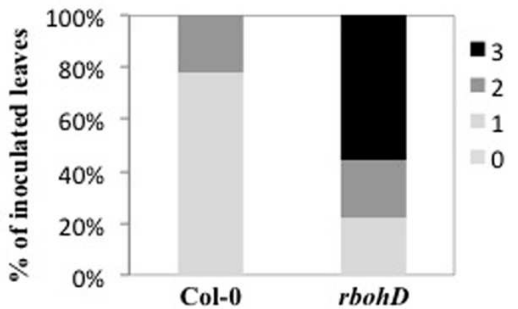

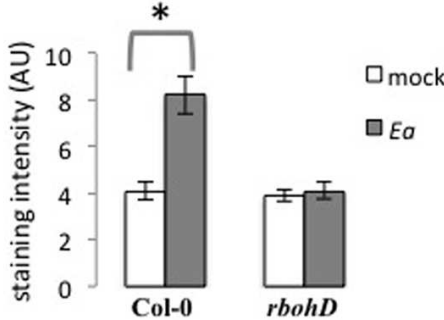

C
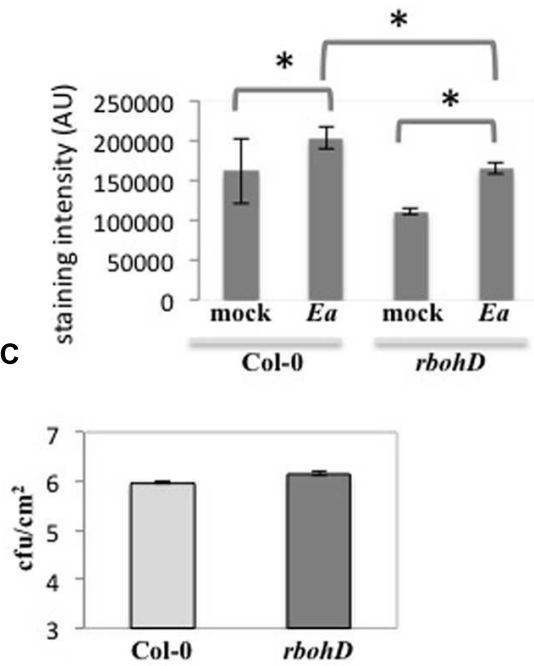

FIGURE $4 \mid$ rbohD is required for intracellular $\mathrm{H}_{2} \mathrm{O}_{2}$ accumulation and limits spread of lesions. (A-C) Leaves of 5-weeks-old plants were inoculated with wild-type $E$. amylovora (Ea) or mock-inoculated (mock) and stained to detect $\mathrm{H}_{2} \mathrm{O}_{2}$ accumulation 18 hpi. (A) Leaves stained with DCFH-DA show intracellular $\mathrm{H}_{2} \mathrm{O}_{2}$ accumulation in green (top), leaves stained with $\mathrm{DAB}$ show $\mathrm{H}_{2} \mathrm{O}_{2}$ accumulation in brown (bottom). The graphs on the right represent mean DCFH-DA intensity (top) and mean DAB staining intensity (bottom), which were determined in 5-10 leaves per condition using the ImageJ Software. The stars indicate a significant difference according to Mann and Whitney's statistical test $(p<0.05)$. (B) Symptom intensity in Col-0 and rbohD mutant 24 hpi. We used a qualitative scale for the intensity of necrosis rating from no necrosis (0) to strong necrosis (3). Pictures corresponding to the scale can be found in Degrave et al. (2008). (C) Bacterial titers in Col-0 and rbohD mutant 24 hpi. No significant difference between titers in Col-0 and rbohD leaves according to Mann and Whitney's statistical test $(p<0.05)$.

the ImageJ software (Figure 3C) and statistical analysis confirms that the level of fluorescence in the 13-1-2 line was the same as the control line whereas E. amylovora-inoculated leaves showed significant levels of fluorescence according to Mann and Whitney's test $(p<0.05)$. These results confirm that DspA/E, when expressed in the plant cell, does not trigger significant intracellular $\mathrm{H}_{2} \mathrm{O}_{2}$ accumulation.

Our results clearly show that DspA/E is required and sufficient for accumulation of $\mathrm{CeCl}_{3}$-detectable ROS in the apoplast but does not trigger significant intracellular ROS.

\section{rbohD is Required for Intracellular $\mathrm{H}_{2} \mathrm{O}_{2}$ Accumulation and Limits Spread of Lesions}

To determine the role of $\mathrm{RbohD}$ in the interaction between A. thaliana and E. amylovora, we measured ROS production in the rbohD knock-out (KO) mutant following $E$. amylovora inoculation (Figure 4). Using DCFH-DA, we did not detect significant fluorescence signal in the rbohD mutant inoculated with E. amylovora (Figure $\mathbf{4 A}$ ) as we did in wild-type Col-0 plants (Figures 1A and 4A). Using DAB staining, which detects $\mathrm{H}_{2} \mathrm{O}_{2}$ in both intra- and intercellular spaces, we found a weak brown staining in the rbohD mutant inoculated with E. amylovora (Figure 4A). The mock-treated leaves of both genotypes displayed no DAB- or DCFH-DA staining, as expected (Figure 4A). DCFH-DA staining intensity was not significantly higher in the rbohD mutant inoculated with E. amylovora compared to mock-treated rbohD plants (Figure 4A). DAB staining intensity was significantly higher in the rbohD mutant inoculated with E. amylovora compared to mock-treated rbohD plants but significantly lower than in wild-type inoculated plants (Figure 4A). These data indicate that RbohD is required for only part of the ROS accumulation that is triggered during the nonhost resistance response of A. thaliana toward E. amylovora, and probably mostly intracellular ROS.

To understand the role of RbohD-dependent ROS accumulation during non-host resistance, we measured necrotic symptom intensity and bacterial titers following inoculation of rbohD mutant plants with E. amylovora (Figure 4). As described previously (Degrave et al., 2008), E. amylovora inoculation of wild-type Col-0 plants triggered necrotic symptoms that 
start to appear $24 \mathrm{hpi}$ and can lead to strong necrosis after several days. In the rbohD mutant, the proportion of leaves with strong necrotic symptoms was significantly higher 24 hpi than in wild-type Col-0 plants (Figure 4B). This indicated that $\mathrm{RbohD}$ had a negative effect on necrosis development in leaves in response to E. amylovora inoculation. In contrast, we did not find any significant effect of the rbohD mutation on E. amylovora bacterial titers (Figure 4C), suggesting either that RbohD-dependent ROS production is not involved in non-host resistance against E. amylovora or that it is not the sole actor of non-host resistance.

$\mathrm{DspA} / \mathrm{E}$ is known to induce cell death in both host and non-host plants. We showed previously that in the 13-1-2 line, induction of DspA/E expression with estradiol reduced seedling growth and ultimately led to the plant's death (Degrave et al., 2013). To determine the effect of RbohD on this toxicity, we constructed a double homozygous line for the rbohD KO mutation and for the 13-1-2 transgene. Following estradiol treatment, the control line (7-2-1) and the rbohD mutant showed no reduction in root growth whereas the 13-1-2 line showed decreased root growth with increasing estradiol concentrations (Figure 5) as expected. Root growth of the double rbohD 13-1-2 line was reduced by estradiol in a similar manner as the 13-12 line (Figure 5). These data show that the rbohD mutation did not suppress DspA/E-induced cell death and toxicity in plant cells.

\section{Co-inoculation of E. amylovora with Cycloheximide (CHX) Reduces Apoplastic But Not Intracellular ROS Accumulation}

We showed previously that co-inoculation of $A$. thaliana leaves with E. amylovora and CHX, an inhibitor of eukaryotic protein synthesis, led to a strong increase in bacterial titers compared to leaves inoculated with E. amylovora alone (Moreau et al., 2012). This led us to hypothesize that CHX co-inoculation suppressed the triggering of plant defense effective against E. amylovora. In order to determine the effect of $\mathrm{CHX}$ on intracellular and apoplastic ROS accumulation during the

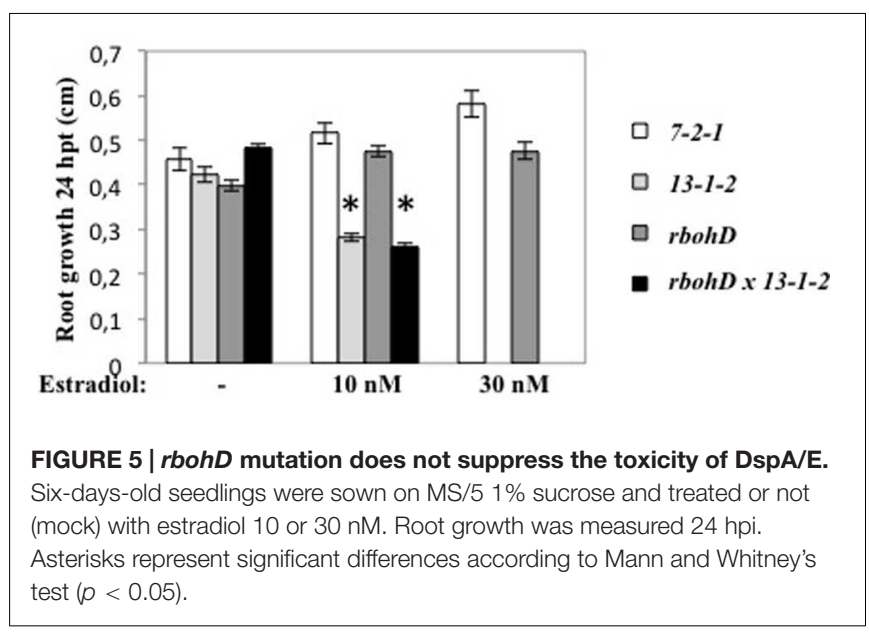

non-host response of $A$. thaliana to E. amylovora, we stained inoculated leaves with DCFH-DA or DAB as described earlier. In mock-inoculated leaves, no DCFH-DA fluorescence could be detected, whether the leaves were inoculated with water alone or with CHX (Figure 6A). In leaves inoculated with E. amylovora, DCFH-DA fluorescence was detected as described earlier but the staining was stronger in leaves co-inoculated with $\mathrm{CHX}$ (Figure 6A). These results indicated that CHX co-inoculation did not repress intracellular ROS accumulation triggered by E. amylovora.

To determine whether apoplastic ROS accumulation was affected by co-inoculation with CHX, we stained E. amylovorainoculated leaves, co-inoculated with $\mathrm{CHX}$ or not, with DAB. The leaves were observed under a microscope to assess tissue localization of the staining as in Figure 1C. No DAB staining was observed in water or CHX-inoculated leaves (not shown). In leaves inoculated with E. amylovora alone, we found intracellular DAB staining as well as staining at the periphery of the cells as expected. In contrast, in leaves co-inoculated with E. amylovora and CHX we found only intracellular $\mathrm{DAB}$ staining and no staining at the periphery of the cells (Figure 6B). These results indicate that $\mathrm{CHX}$ represses the apoplastic ROS accumulation that is triggered by E. amylovora in A. thaliana.

\section{DISCUSSION}

Erwinia amylovora is a pathogenic bacterium with a narrow host range and the model plants $A$. thaliana and $N$. benthamiana are not considered as hosts for this bacterium. Indeed, in both cases the bacterium is able to multiply transiently in leaves after which bacterial multiplication is repressed by nonhost resistance (Oh et al., 2007; Degrave et al., 2008). We showed previously that in A. thaliana, this non-host resistance is associated with the induction of several lines of defense including ROS accumulation (Moreau et al., 2012). ROS accumulation during plant pathogen interactions is generally associated with defense, in particular in the context of non-host resistance. In the case of E. amylovora, it has been suggested that ROS accumulation is critical for cell death activation and for disease, although this has not been formally demonstrated (Venisse et al., 2003). Thus, to better understand the role of ROS accumulation observed in A. thaliana leaves infected with E. amylovora, we investigated in detail the localization of this ROS accumulation and the role of the major T3E of E. amylovora, DspA/E, in this process.

Using a combination of ROS detection probes, we show in the present work that $\mathrm{H}_{2} \mathrm{O}_{2}$ accumulated both in the cytoplasm and in the apoplast in response to infection with E. amylovora. Moreover, TEM analysis showed that $\mathrm{H}_{2} \mathrm{O}_{2}$ accumulated in the cytosol and in several organelles, including mitochondria, peroxisomes and chloroplasts, as well as in the apoplast. Previous reports have shown similar localization of ROS accumulation during non-host interactions. For example, inoculation of lettuce with $P$. syringae pv. phaseolicola resulted in hydrogen peroxide accumulation in the apoplast (Bestwick et al., 1997) while 


\section{A}

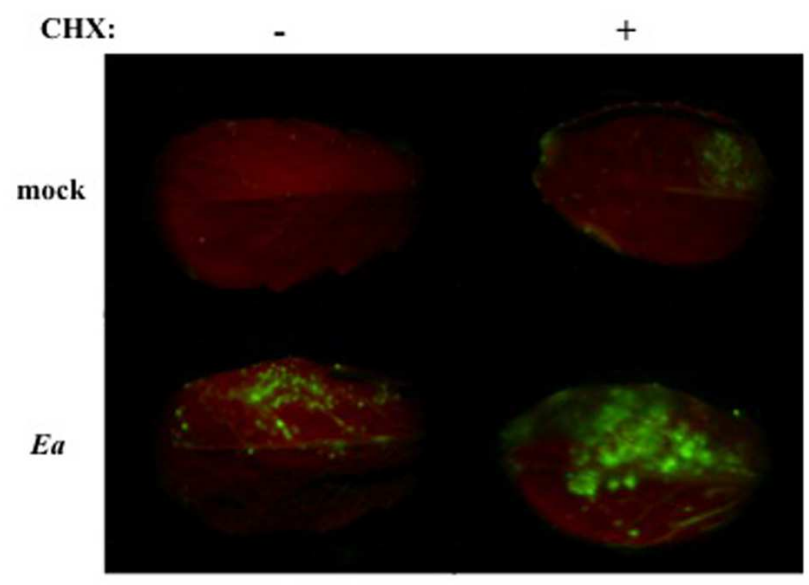

B

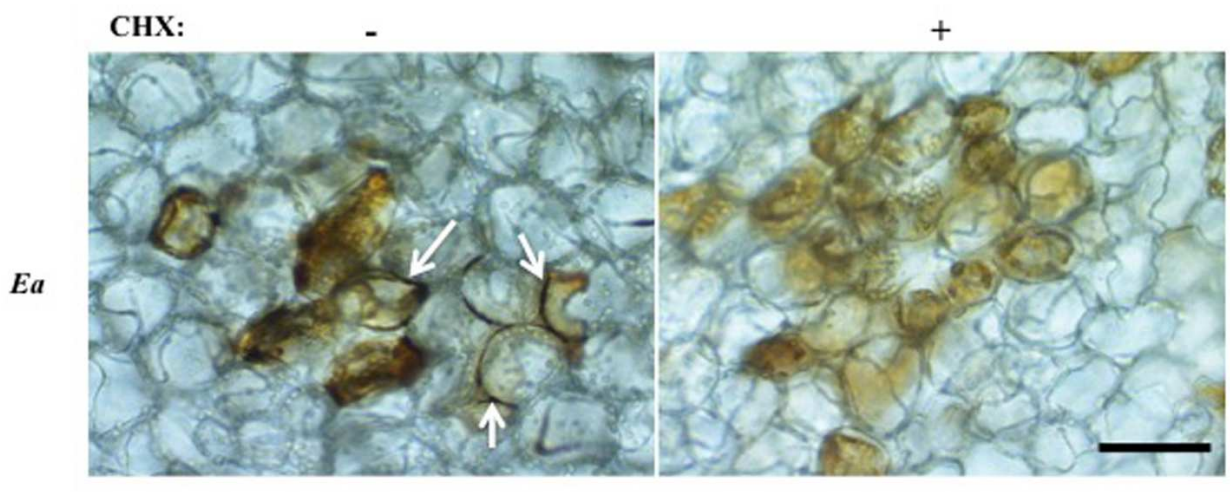

FIGURE 6 | Co-inoculation of E. amylovora with $\mathrm{CHX}$ reduces apoplastic ROS accumulation. (A,B) Leaves of 5-weeks-old plants were mock-treated (mock) or inoculated with wild-type E. amylovora (Ea) and co-inoculated (+) or not (-) with $4 \mu \mathrm{g} / \mathrm{ml}$ cycloheximide. Leaves were stained with DCFH-DA or DAB to detect $\mathrm{H}_{2} \mathrm{O}_{2}$ accumulation 18 hpi. (A) Only the top half of the leaf blade was inoculated. Leaves stained with DCFH-DA show intracellular $\mathrm{H}_{2} \mathrm{O}_{2}$ accumulation in green.

(B) Leaves stained with $\mathrm{DAB}$ show $\mathrm{H}_{2} \mathrm{O}_{2}$ accumulation in brown. Arrows indicate DAB staining in the apoplast. Bar: $50 \mu \mathrm{m}$.

inoculation of tobacco with $X$. campestris pv. vesicatoria led to hydrogen peroxide accumulation in the chloroplasts (Zurbriggen et al., 2009). Although, the specific role of the different sources of ROS in plant pathogen interactions is still not fully understood, ROS accumulation in mitochondria and chloroplasts are strongly linked to the triggering of cell death (Van Akent and Van Breusegem, 2015). Furthermore, it is now clear that ROS are more than deleterious molecules causing oxidative stress and that the spatiotemporal control of ROS production plays an important role in the specificity of ROS signaling (Mignolet-Spruyt et al., 2016). In particular, apoplastic ROS plays a role in both longdistance extracellular signaling and initiation of intracellular signaling.

Our data show that RbohD strongly contributes to $\mathrm{H}_{2} \mathrm{O}_{2}$ accumulation in response to $E$. amylovora inoculation in A. thaliana. The role of Rboh-dependent ROS in plant pathogen interactions is not clear as the corresponding rboh mutants show reduced, normal or increased cell death depending on the initial cell death trigger (Van Akent and Van Breusegem, 2015). However, we found that the rbohD mutation did not suppress
E. amylovora-triggered necrosis or DspA/E toxicity. These results indicate that in $A$. thaliana DspA/E-triggered cell death does not require $\mathrm{RbohD}$-dependent $\mathrm{H}_{2} \mathrm{O}_{2}$ accumulation. In contrast, we found that RbohD-dependent $\mathrm{H}_{2} \mathrm{O}_{2}$ accumulation was important for the restriction of necrosis since rbohD mutant plants showed necrotic symptoms of higher intensity than wild-type plants. Previous work indicated a role for $\mathrm{RbohD}$ in the limitation of spreading lesions (Torres et al., 2005), consistent with our observations.

We have previously shown that $\mathrm{CHX}$, an inhibitor of eukaryotic protein synthesis, strongly increases E. amylovora bacterial titers in $A$. thaliana. Co-inoculation with $\mathrm{CHX}$ suppressed callose deposition (Moreau et al., 2012), a cellwall based defense normally suppressed during disease in host plants (DebRoy et al., 2004). However, the callose-deficient pmr4 mutant did not allow an increase in bacterial multiplication, indicating that suppression of callose deposition was, at least not exclusively, the means by which $\mathrm{CHX}$ allowed E. amylovora multiplication (Moreau et al., 2012). Here we show that coinoculation of E. amylovora with $\mathrm{CHX}$ did not suppress 
A

\begin{tabular}{|c|c|c|c|c|}
\hline genotype & Col-0 & Col-0 & Col-0 & Col-0 \\
\hline inoculum & $E a$ & $d s p A / E$ & $d s p A / E+\mathrm{CHX}$ & $E a+C H X$ \\
\hline apoplastic ROS & yes & no & no & no \\
\hline intracellular ROS & yes & yes & yes & yes \\
\hline bacterial titer & + & - & + & +++ \\
\hline reference & this work and [9] & this work and [9] & {$[10]$} & this work and [16] \\
\hline B & & & oplastic & \\
\hline
\end{tabular}

FIGURE 7 | Overview of Arabidopsis thaliana non-host resistance against $\mathbf{E}$. amylovora. (A) Overview of ROS production and bacterial growth in the different combinations of inoculum and genotype. Depending on the combination, ROS accumulate in the apoplast and/or in the cytoplasm or don't accumulate. The combinations lead, $24 \mathrm{hpi}$, to either a rapid decrease in bacterial titers $(-)$, a weak and transient increase $(+)$ or a strong increase in bacterial titer (+++). (B) Model of $A$. thaliana non-host resistance against $E$. amylovora. The T3E DspAVE and other elements trigger apoplastic ROS and other defenses that can be repressed by co-inoculation with $\mathrm{CHX}$. CHX: cycloheximide, Dsp: DspA/E, Ea: E. amylovora, ap: apoplast, cy: cytoplasm, RPL: ribosomal protein, dashed lines: defense that reduces bacterial titers, continuous lines: defense repression that increases bacterial titers. Numbers in between brackets indicate the reference of the corresponding work.

intracellular ROS accumulation (Figure 6A) but strongly reduced apoplastic ROS accumulation (Figure 6B). These data suggest that apoplastic ROS is important for non-host resistance and that intracellular ROS is not. Thus, apoplastic ROS and callose deposition could constitute additive layers of non-host resistance, efficient against E. amylovora and both suppressed by $\mathrm{CHX}$ (Figure 7B).

$\mathrm{DspA} / \mathrm{E}$ is the major $\mathrm{T} 3 \mathrm{E}$ of $E$. amylovora and, in host plants, is considered to be the main trigger of ROS production (Venisse et al., 2003). However, these conclusions are based on the indirect observation that DspA/E leads to an increase in ROS detoxification activity. Our results show that, in A. thaliana, a $d s p A / E$-deficient strain is able to induce intracellular ROS accumulation but not apoplastic ROS (Figure 7A). These data suggest either that DspA/E is not involved in intracellular ROS triggering in the non-host context or that it plays a redundant role with another effector as suggested from data obtained on host plants (Venisse et al., 2003). Concerning apoplastic ROS, our data clearly indicate a role for $\mathrm{DspA} / \mathrm{E}$ in the triggering of its accumulation in the non-host context. Thus our data demonstrate a specific role for the T3E DspA/E in the triggering of apoplastic ROS. Apoplastic peroxidases are known to play a role in ROS production in response to pathogens (Bindschedler et al., 2006). One could hypothesize that DspA/E increases apoplastic peroxidase activity either trough direct targeting or following an induction of their expression at the transcriptomic level. Indeed, our previous transcriptome analysis (Degrave et al., 2013) showed that expression of DspA/E in planta led to an increased expression of $P R X 52$, a peroxidase that accumulates in the apoplast in A. thaliana leaves infected with the fungus Verticillium longisporum (Floerl et al., 2012). Furthermore, peroxidases have been shown to be involved in resistance, which is consistent with our observations that apoplastic ROS is correlated with non-host resistance to E. amylovora. For example, prx33 KO mutants show a clear increase in susceptibility to $P$. syringae (Daudi et al., 2012). Further investigation is required to determine whether apoplastic peroxidases are indeed involved in DspA/E-dependent ROS accumulation and non-host resistance to E. amylovora.

Although, DspA/E triggers non-host resistance-associated apoplastic ROS detected by $\mathrm{CeCl}_{3}$, our previous data show that DspA/E contributes to the weak bacterial multiplication observed in the non-host context (Degrave et al., 2013; Figure 7A). This is consistent with previous work on $N$. benthamiana that indicated a dual role for DspA/E in the non-host context (Oh et al., 2007). This suggests either that in the non-host context, DspA/E is required to induce a susceptibility factor or to repress other defenses, albeit inefficiently. Interestingly, we showed previously 
that DspA/E, which localizes in the nucleolus, represses protein synthesis and represses the expression of ribosomalprotein (RPL) genes (Degrave et al., 2013). In particular, we have found that expression of RPL19 was repressed in the 13-1-2 line when DspA/E expression was induced by estradiol (data not shown). Considering the recent results presented by Nagaraj et al. (2015), one could hypothesize that DspA/E targets RPL19 to suppress protein synthesis and thus defense, but that this process is inefficient in non-host plants (Figure 7B).

Altogether, our results show that E. amylovora triggers both intracellular and apoplastic ROS accumulation (Figure 7A). However, consistent with the key role of the apoplast in plant defense (Gupta et al., 2015), only apoplastic ROS accumulation was found to be correlated with non-host resistance. Nonhost resistance is generally thought to be a multilayer process that leads to durable resistance because it is more difficult for a pathogen to break than gene-for-gene resistance (SenthilKumar and Mysore, 2013). Thus, identifying the molecular actors and understanding the mechanisms of non-host resistance will contribute to the definition of new strategies to develop broadspectrum durable resistance.

\section{REFERENCES}

Asai, S., and Yoshioka, H. (2009). Nitric oxide as a partner of reactive oxygen species participates in disease resistance to necrotrophic pathogen Botrytis cinerea in Nicotiana benthamiana. Mol. Plant Microbe Interact. 22, 619-629. doi: 10.1094/MPMI-22-6-0619

Azevedo, H., Lino-Neto, T., and Tavares, R. M. (2008). The necrotroph Botrytis cinerea induces a non-host type II resistance mechanism in Pinus pinaster suspension-cultured cells. Plant Cell Physiol. 49, 386-395. doi: 10.1093/pcp/pcn015

Barny, M., Guinebretière, M., Marçais, B., Coissac, E., Paulin, J., and Laurent, J. (1990). Cloning of a large gene cluster involved in Erwinia amylovora CFBP1430 virulence. Mol. Microbiol. 4, 777-786. doi: 10.1111/j.13652958.1990.tb00648.x

Barny, M. A., Boureau, T., Degrave, A., Fagard, M., Bouteau, F., Gaubert, S., et al. (2008). "Type III effectors of E. amylovora: synergistic and antagonistic effects," in Proceedings of the Eleventh International Workshop on Fire Blight, eds K. B. Johnson and V. O. Stockwell (Leuven: International Society for Horticultural Science), 215-220.

Bestwick, C. S., Brown, I. R., Bennett, M. H. R., and Mansfield, J. W. (1997). Localization of hydrogen peroxide accumulation during the hypersensitive reaction of lettuce cells to Pseudomonas syringae pv phaseolicola. Plant Cell 9, 209-221. doi: 10.1105/tpc.9.2.209

Bindschedler, L. V., Dewdney, J. K., Blee, A. J., Stone, M., Asai, T., Plotnikov, J., et al. (2006). Peroxidase-dependent apoplastic oxidative burst in Arabidopsis required for pathogen resistance. Plant J. 47, 851-863. doi: 10.1111/j.1365313X.2006.02837.x

Daudi, A., Cheng, Z., O’Brien, J., Mammarella, N., Khan, S., Ausubel, F., et al. (2012). The apoplastic oxidative burst peroxidase in Arabidopsis is a major component of pattern-triggered immunity. Plant Cell 24, 275-287. doi: 10.1105/tpc.111.093039

DebRoy, S., Thilmony, R., Kwack, Y., Nomura, K., and He, S. (2004). A family of conserved bacterial effectors inhibits salicylic acidmediated basal immunity and promotes disease necrosis in plants. Proc. Natl. Acad. Sci. U.S.A. 101, 9927-9932. doi: 10.1073/pnas.04016 01101

Degrave, A., Fagard, M., Perino, C., Brisset, M. N., Gaubert, S., Laroche, S., et al. (2008). Erwinia amylovora type three-secreted proteins trigger cell death and defense responses in Arabidopsis thaliana. Mol. Plant Microbe Interact. 21, 1076-1086. doi: 10.1094/MPMI-21-8-1076

\section{AUTHOR CONTRIBUTIONS}

$\mathrm{AL}$ and MF wrote the manuscript; $\mathrm{AL}, \mathrm{OP}, \mathrm{EW}$, and $\mathrm{MF}$ performed the experiments.

\section{FUNDING}

AL was supported by a grant from the French Ministry for Research and Education (MENRT). We thank the ANR for funding (ANR JCJC DspCellDeath) and the SPS labex for financial support to the IJPB.

\section{ACKNOWLEDGMENTS}

We thank H. Ferry for taking excellent care of our plants, J. Stellingwerf and A. Mai for technical assistance, G. Toutirais for support with the TEM and the electronic microscopy department of Institut de Biologie Paris-Seine. We thank MC Soulié, A. Krapp, D. Expert, and T. Elmayan for support and suggestions.

Degrave, A., Moreau, M., Launay, A., Barny, M.-A., Brisset, M.-N., Patrit, O., et al. (2013). The bacterial effector DspA/E is toxic in Arabidopsis thaliana and is required for multiplication and survival of fire blight pathogen. Mol. Plant Pathol. 14, 506-517. doi: 10.1111/mpp.12022

Fagard, M., Dellagi, A., Roux, C., Perino, C., Rigault, M., Boucher, V., et al. (2007). Arabidopsis thaliana expresses multiple lines of defense to counterattack Erwinia chrysanthemi. Mol. Plant Microbe Interact 20, 794-805. doi: 10.1094/MPMI-20-7-0794

Floerl, S., Majcherczyk, A., Possienke, M., Feussner, K., Tappe, H., Gatz, C., et al. (2012). Verticillium longisporum infection affects the leaf apoplastic proteome, metabolome, and cell wall properties in Arabidopsis thaliana. PLoS ONE 7:e31435. doi: 10.1371/journal.pone.0031435

Gupta, R. S., Lee, E. G., Agrawal, K., Rakwal, R., Park, S., Wang, Y., et al. (2015). Understanding the plant-pathogen interactions in the context of proteomics-generated apoplastic proteins inventory. Front. Plant Sci. 6:352. doi: 10.3389/fpls.2015.00352

Lamb, C., and Dixon, R. (1997). The oxidative burst in plant disease resistance. Annu. Rev. Plant Physiol. Plant Mol. Biol. 48, 251-275. doi: 10.1146/annurev.arplant.48.1.251

Marino, D., Dunand, C., Puppo, A., and Pauly, N. (2012). A burst of plant NADPH oxidases. Trends Plant Sci. 17, 9-15. doi: 10.1016/j.tplants.2011. 10.001

Mignolet-Spruyt, L., Xu, E., Idänheimo, N., Hoeberichts, F. A., Mühlenbock, P., Brosché, M., et al. (2016). Spreading the news: subcellular and organellar reactive oxygen species production and signaling. J. Exp. Bot. doi: 10.1093/jxb/erw080 [Epub ahead of print].

Moreau, M., Degrave, A., Vedel, R., Bitton, F., Patrit, O., Renou, J.-P., et al. (2012). EDS1 Contributes to nonhost resistance of Arabidopsis thaliana against Erwinia amylovora. Mol. Plant Microbe Interact. 25, 421-430. doi: 10.1094/MPMI-0511-0111

Nagaraj, S., Senthil-Kumar, M., Ramu, V. S., Wang, K., and Mysore, K. S. (2015). Plant ribosomal proteins, RPL12 and RPL19, play a role in nonhost disease resistance against bacterial pathogens. Front. Plant Sci. 6:1192. doi: 10.3389/fpls.2015.01192

Oh, C., Martin, G., and Beer, S. (2007). DspA/E, a type III effector of Erwinia amylovora, is required for early rapid growth in Nicotiana benthamiana and causes NbSGT1-dependent cell death. Mol. Plant Pathol. 8, 255-265. doi: 10.1111/j.1364-3703.2007.00390.x

Rojas, M., Senthil-Kumar, M., Wang, K. C., Ryu, M., Kaundal, A., and Mysore, K. S. (2012). Glycolate oxidase modulates reactive oxygen species-mediated 
signal transduction during nonhost resistance in Nicotiana benthamiana and Arabidopsis. Plant Cell 24, 336-352. doi: 10.1105/tpc.111.093245

Schulze-Lefert, P., and Panstruga, R. (2011). A molecular evolutionary concept connecting nonhost resistance, pathogen host range, and pathogen speciation. Trends Plant Sci. 16, 117-125. doi: 10.1016/j.tplants.2011.01.001

Senthil-Kumar, M., and Mysore, K. S. (2013). Nonhost resistance against bacterial pathogens: retrospectives and prospects. Annu. Rev. Phytopathol. 51:407. doi: 10.1146/annurev-phyto-082712-102319

Torres, M., Dangl, J., and Jones, J. (2002). Arabidopsis gp91phox homologues AtrbohD and AtrbohF are required for accumulation of reactive oxygen intermediates in the plant defense response. Proc. Natl. Acad. Sci. U.S.A. 99, 517-522. doi: 10.1073/pnas.012452499

Torres, M. A., Jones, J. D. G., and Dangl, J. L. (2005). Pathogen-induced, $\mathrm{NADPH}$ oxidase-derived reactive oxygen intermediates suppress spread of cell death in Arabidopsis thaliana. Nat. Genet. 37, 1130-1134. doi: 10.1038/ ng1639

Van Akent, O., and Van Breusegem, F. (2015). Licensed to kill: mitochondria, chloroplasts, and cell death. Trends Plant Sci. 20, 754-766. doi: 10.1016/j.tplants.2015.08.002

van Baarlen, P., Woltering, E., Staats, M., and van Kan, J. (2007). Histochemical and genetic analysis of host and non-host interactions of Arabidopsis with three Botrytis species: an important role for cell death control. Mol. Plant Pathol. 8, 41-54. doi: 10.1111/j.1364-3703.2006.00367.x
Venisse, J., Barny, M., Paulin, J., and Brisset, M. (2003). Involvement of three pathogenicity factors of Erwinia amylovora in the oxidative stress associated with compatible interaction in pear. FEBS Lett. 537, 198-202. doi: 10.1016/S0014-5793(03)00123-6

Venisse, J., Gullner, G., and Brisset, M. (2001). Evidence for the involvement of an oxidative stress in the initiation of infection of pear by Erwinia amylovora. Plant Physiol. 125, 2164-2172. doi: 10.1104/pp.125.4.2164

Zurbriggen, M. D., Carrillo, N., Tognetti, V. B., Melzer, M., Peisker, M., Hause, B., et al. (2009). Chloroplast-generated reactive oxygen species play a major role in localized cell death during the non-host interaction between tobacco and Xanthomonas campestris pv. vesicatoria. Plant J. 60, 962-973. doi: 10.1111/j.1365-313X.2009.04010.x

Conflict of Interest Statement: The authors declare that the research was conducted in the absence of any commercial or financial relationships that could be construed as a potential conflict of interest.

Copyright (c) 2016 Launay, Patrit, Wénès and Fagard. This is an open-access article distributed under the terms of the Creative Commons Attribution License (CC BY). The use, distribution or reproduction in other forums is permitted, provided the original author(s) or licensor are credited and that the original publication in this journal is cited, in accordance with accepted academic practice. No use, distribution or reproduction is permitted which does not comply with these terms. 